\title{
Properties and Thermal Phase Behaviour of Binary Mixtures of Homologues of Unsymmetrically Substituted Phenyl Benzoates
}

\author{
M. Roushdy \\ Department of Physics, Faculty of Science, \\ University of Cairo, Giza, Egypt
}

Sixteen liquid crystalline esters of the type 4- $\left(n-C_{n} \mathrm{H}_{2 n+1} \mathrm{O}\right) \mathrm{C}_{6} \mathrm{H}_{4}$ $\mathrm{COOC}_{6} \mathrm{H}_{4} \mathrm{X}-4$ have been investigated for their thermal phase behaviour. These compounds constitute four homologous series that differ from each other by the substituent $\mathrm{X}$. The latter varies between $\mathrm{CH}_{3} \mathrm{O}, \mathrm{CH}_{3}, \mathrm{CN}$, and $\mathrm{NO}_{2}$. The number (n) of carbon varies, within a homologous series, between 6, 8, 14, and 16. All possible binary mixtures made from any two homologues that cover the whole composition range were prepared and characterized for their mesophase behaviour by differential scanning calorimetry (DSC) and polarizing light microscopy. Twenty four phase diagrams for the various binary combinations were constructed (i) to investigate the dependence of the phase behaviour of mixed systems upon differences in the length of the alkoxy group, (ii) to determine the eutectic composition, and its phase transitions, in each system. Each eutectic system of the binary mixtures (I6/In)X was investigated for its birefringence dependency on temperature via measurement of its refractive index as a function of temperature. The estimated orientational order parameter $S$ for each system was used to examine the effect of the differences in chain length of alkoxy group on its optical properties.

\section{Introduction:}

Interests in liquid crystalline materials have expanded greatly in recent years because of their important role in new and emerging electro-optical technologies and their richness in physical phenomena. Liquid crystal materials for device applications are mostly mixtures, usually of eutectic composition.

In general, binary mesophase systems exhibit eutectic behaviour in their solid-mesophase transition temperatures $\left(\mathrm{T}_{\mathrm{m}}\right)$, whereas their mesophaseisotropic transition temperatures $\left(T_{c}\right)$ vary linearly with composition [1]. However, non-linear $\mathrm{T}_{\mathrm{c}}$-composition behaviours have been reported for some 
mixtures when one component was chloro [2] or nitro [2, 3] substituted liquid crystals. In most cases, a mixed mesomorphic system is employed in order to depress the melting point $\left(\mathrm{T}_{\mathrm{m}}\right)$ of one, or both, of the pure components so as to obtain its mesophase over a more readily accessible temperature range. In previous works [4-7], mixtures were made from: (i) components of the same core structures and of similar alkoxy chain length $(\mathrm{R})$ but of different polar substituents (X) [4, 5], (ii) components of the same core structure and polar substituent (X) but of different alkoxy chain length (R) [6], and (iii) either components are of different core structure but bearing the same grouping (X and R) [7]. In these studies it has been found that: for the long core structure $\mathrm{C}_{6} \mathrm{H}_{4} \mathrm{COOC}_{6} \mathrm{H}_{4} \mathrm{OOCC}_{6} \mathrm{H}_{4}$ - binary phase diagrams showed eutectic behaviour in case (i), i.e. for components bearing substituents (X) of different polarity, while in case (ii), i.e. of components bearing alkoxy groups with different chain length, a regular change of $\mathrm{T}_{\mathrm{m}}$ with composition was observed especially for $\mathrm{X}=\mathrm{CH}_{3} \mathrm{O}, \mathrm{Cl}, \mathrm{NO}_{2}$, and $\mathrm{CN}$. For another extended core structure, namely, $\mathrm{C}_{6} \mathrm{H}_{4} \mathrm{COO}-\mathrm{C}_{6} \mathrm{H}_{4}-\mathrm{N}=\mathrm{N}-\mathrm{C}_{6} \mathrm{H}_{4}$, the phase diagrams of case (i) almost showed regular change with composition of the melting points $\left(\mathrm{T}_{\mathrm{m}}\right)$ especially for longer chain length and polar groups with similar electronic nature. Case (iii) was investigated using components from the two previously mentioned cores but bearing the same R and X. In most of the cases, phase diagrams showed eutectic behaviour independent of the type of $\mathrm{X}$ or length of $\mathrm{R}$. However, their eutectic mixtures were found to melt at temperature greater than $70{ }^{\circ} \mathrm{C}$. These results have encouraged to use, in the present investigation, the simpler short core, namely $-\mathrm{C}_{6} \mathrm{H}_{4} \mathrm{COOC}_{6} \mathrm{H}_{4}$ - (I) because of the low melting points of their eutectic composition.

While intensive studies concerning the optical behaviour of the single components constituting the three above cases (i), (ii), and (iii) have been recently presented [8-13], a little has been observed for binary mixtures [13-16]. This reflects the importance of presenting systematic investigations on the optical behaviour of binary mixtures especially at their eutectic composition. In this respect, the effect of the polar subtituents on the optical parameters of binary mixtures of compounds of the type I has been reported [16].

The object of this work is to investigate the effect of the difference in the alkoxy chain length on the optical properties and thermal mesophase behaviour of binary mixtures made from any two homologues of I bearing the same substituent $\mathrm{X}$. 


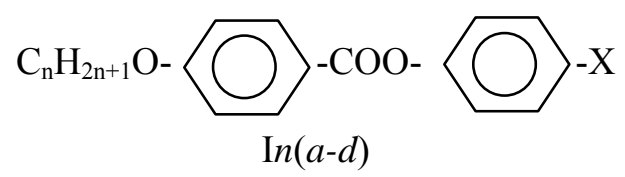

$\mathrm{I} 6, n=6 ; \mathrm{I} 8, n=8 ; \mathrm{I} 14, n=14 ; \mathrm{I} 16, n=16$

$a, \mathrm{X}=\mathrm{CH}_{3} \mathrm{O} ; b, \mathrm{X}=\mathrm{CH}_{3} ; c, \mathrm{X}=\mathrm{CN} ; d, \mathrm{X}=\mathrm{NO}_{2}$

\section{Experimental}

\subsection{Preparation of materials}

The pure components $\operatorname{In}(a-d)$ used in the preparation of the investigated mixtures were kindly supplied by Prof. Dr. M. M. Naoum, Chemistry Department, Faculty of Science, University of Cairo, where they were prepared according to the method previously described [17].

\subsection{Physical characterization}

Calorimetric investigation was made using a Polymer Laboratories (England) differential scanning calorimeter, PL DSC, with nitrogen as a purge gas. The instrument was calibrated from the melting points of ultra-pure metals, using a value of $28.45 \mathrm{Jg}^{-1}$ for the enthalpy of fusion of indium. Typical heating rate was $10 \mathrm{Kmin}^{-1}$, with a mass of 2-3 $\mathrm{mg}$. In the phase diagrams the symbols 0 denote solid-mesophase or solid-isotropic transitions (on heating), $\square$ denote mesophase-isotropic transitions (on heating or cooling) and $\bullet$ denote isotropicmesophase transitions (on cooling).

\subsection{Refractive index measurements}

Measurements of the refractive index as a function of temperature were performed using an Abbe 60 refractometer attached to an ultra-thermostat. The respective accuracy in refractive index and temperature measurements were \pm 0.0001 and $\pm 0.2{ }^{\circ} \mathrm{C}$. The investigated samples were used without any external aligning aids. A polarizer placed over the eyepiece allowed a distinct separation of the dark and bright space in the eyepiece. The refractive indices were measured as a function of temperature during cooling the isotropic phase down to the nematic one. Refractive indices at temperatures other than $20{ }^{\circ} \mathrm{C}$ were corrected in order to compensate for the change of refractive index of the prism brought about by the working temperature, since the instrument has been initially calibrated for $20^{\circ} \mathrm{C}$. The correction amounts to 0.0000078 per ${ }^{\circ} \mathrm{C}$.

While the ordinary refractive index, $\mathrm{n}_{0}$, in the nematic phase has been directly measured, the extraordinary refractive index, $\mathrm{n}_{\mathrm{e}}$, was too high to be 
measured by the available refractometer. Thus, $n_{e}$ at each temperature was calculated from the relation [18]:

$$
\mathrm{n}^{2}=(1 / 3)\left(\mathrm{n}_{\mathrm{e}}^{2}+2 \mathrm{n}_{\mathrm{o}}^{2}\right)
$$

where, $\mathrm{n}$ is the extrapolated average refractive index (within the nematic phase) as obtained by the extrapolation of $n_{\text {iso }}$ to the desired temperature.

\section{Results And Discussion:}

\subsection{The thermal behaviour}

\subsubsection{Binary mixtures of homologues bearing an electron-releasing substituent}

Figure (1a-f) represents the phase diagrams for the six possible binary combinations made from the four methoxy-substituted homologues I6a-I16a. Irrespective of the differences between the alkoxy chain lengths of the two components in any binary system, all mixtures investigated were shown to possess eutectic composition. This finding is in contrast with that observed for the corresponding phase diagrams for homologues of the more elongated core structure, $\mathrm{C}_{\mathrm{n}} \mathrm{H}_{2 \mathrm{n}+1} \mathrm{O} \quad \mathrm{C}_{6} \mathrm{H}_{4}-\mathrm{COOC}_{6} \mathrm{H}_{4}-\mathrm{OOCC}_{6} \mathrm{H}_{4}-\mathrm{X}$ (II), where a regular $\mathrm{T}_{\mathrm{m}}$-composition dependences were observed [6] irrespective of the alkoxy chain length. Such discrepancy may be attributed, in the latter case, to the strong intermolecular forces within the solid which is strong enough to be significantly influenced by chain length giving rise to components of similar crystalline structure and of comparable molecular forces of association. This influence is contrasted by the low melting points of pure components of I and of their eutectic mixtures compared with those of II.

The corresponding binary phase diagrams of the homologous series of the methyl substituted homologues $\mathrm{I} b$ are represented graphically in Fig. (2a-f). Since the electron-donating $\mathrm{CH}_{3}$ group is of similar nature to the $\mathrm{CH}_{3} \mathrm{O}$ one, methylsubstituted homologues were expected to give similar binary phase behaviour to those of series I $a$. This was found to be the case; all binary mixtures of homologues of $\mathrm{I} b$ possess eutectic behaviour. Since the methyl group is weak electron-donating, compared with the methoxy one, the methyl-substituted analogues are of lower melting points than the corresponding methoxy-substituted analogues and the melting point of their eutectic mixtures is much more depressed in series $\mathrm{I} b$. This behaviour has led to the appearance of a wider composition-range of nematic phase. It can be further noticed from Figs. ( 1 and 2 ) that while all pure components show their nematic phase monotropically, i.e only on cooling, their eutectic mixtures in some cases, especially in series $\mathrm{I} b$, showed their nematic phases enantiotropically. 




Fig. (1): Binary phase diagrams for mixtures of the methoxy-substituted homologues.
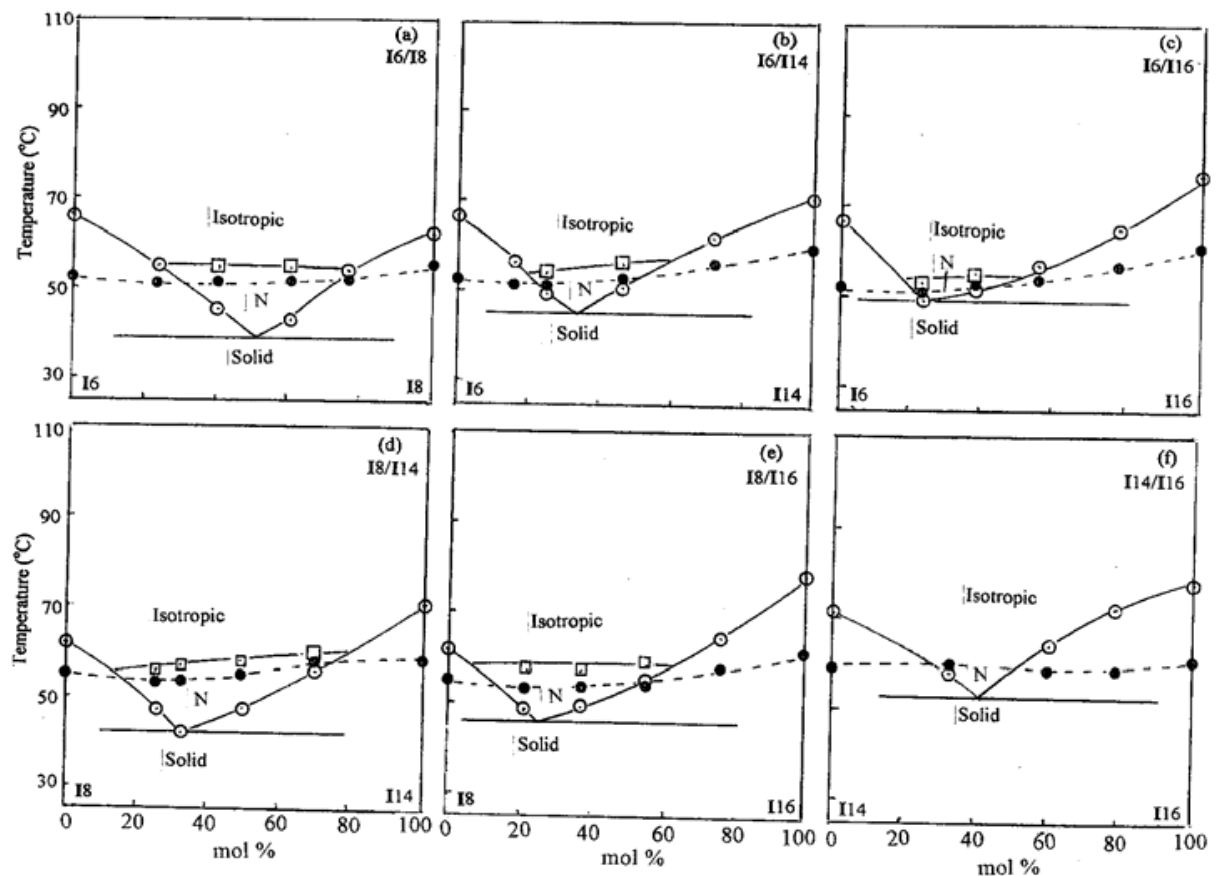

Fig. (2): Binary phase diagrams for mixtures of the methyl-substituted homologues. 


\subsubsection{Binary mixtures of homologues bearing an electron-withdrawing} substituent

The six possible binary phase diagrams, made from the cyanosubstituted homologues (Ic) are represented collectively in Fig. 3(a-f). Since I6c and $I 8 c$ show only the nematic phase enantiotropically while the higher homologues $\mathrm{I} 14 c$ and $\mathrm{I} 16 c$ are enantiotropically smectogenic, the I6c/I8c binary mixtures shows only the nematic phase, whereas the smectic phase starts to appear from the I6c/I14c mixtures and increases through the other mixtures $\mathrm{I} 6 c / \mathrm{I} 16 c, \mathrm{I} 8 c / \mathrm{I} 14 c, \mathrm{I} 8 c / \mathrm{I} 16 c$ to cover the whole composition range in the $\mathrm{I} 14 c / \mathrm{I} 16 c$ mixture.


Fig. (3): Binary phase diagrams for mixtures of the cyano-substituted homologues.

The other electron-withdrawing group, i.e. the $\mathrm{NO}_{2}$, affects the binary phase behaviour to a different extent, in favour of the smectic-A formation, see Fig. (4a-f). Since the lower homologues I6d is monotropically nematogenic while I $8 d$ possesses both the smectic-A and nematic phases, the mixtures containing either one behave differently dependent on the other component. Thus, in mixtures containing I6d, the mixture I6d/I8d showed a wide nematic range $(\approx 20-100 \mathrm{~mol} \%$ I $8 d)$ whereas the smectic phase of I8d disappears 
completely by the addition of more than $25 \mathrm{~mol} \%$ of I6d. The other two systems I6d/I14d and I6d/I16d possess the smectic-A phase starting from about $20 \mathrm{~mol} \%$ of I14d or I16d, respectively. The other extreme appears in the I14d/I16d mixture where the whole composition range is purely smectogenic. The remaining two mixtures containing the homologues I8d, i.e. I8d/I14d and I8d/I16d, show small nematic range $(\approx 0-20$ and $0-40 \mathrm{~mol} \%$ of I14d and I16d, respectively).
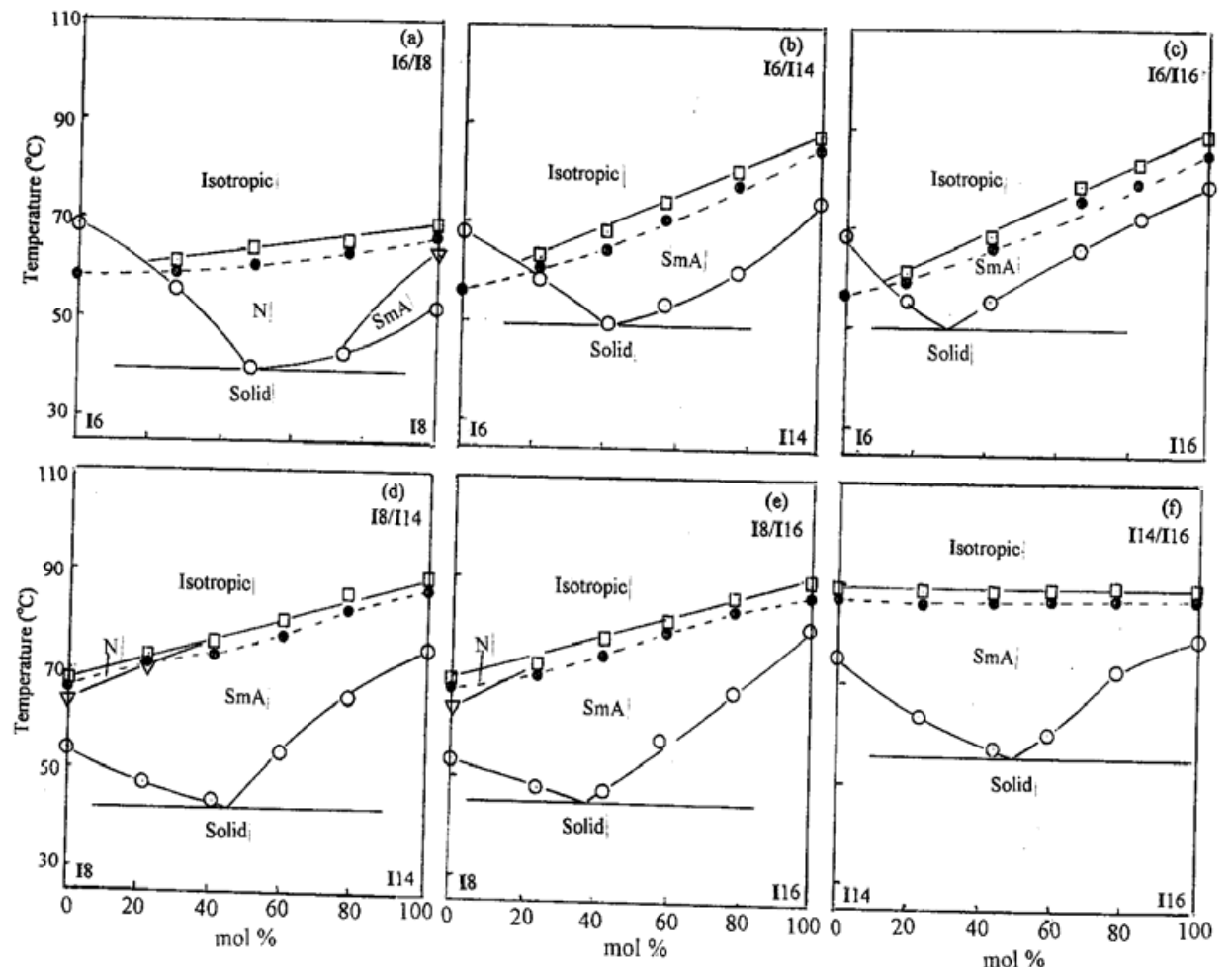

Fig. (4): Binary phase diagrams for mixtures of the nitro-substituted homologues.

\subsection{Optical Properties}

The refractive indices in the isotropic phase as well as in the mesophase were measured as function of temperature for each binary mixture at its eutectic composition. The mole percent of the mixtures at their eutectic composition and their transition temperatures are listed in Table (1). The measured values of $n_{o}$ both with the deduced and the calculated ones of $n$ and $n_{e}$ respectively, have been used to estimate $\delta . S$, using the method of Vuks [19], from the equation: 


$$
\delta . S=\frac{1}{3}\left(\frac{\mathrm{n}_{\mathrm{e}}^{2}-\mathrm{n}_{\mathrm{o}}^{2}}{\mathrm{n}^{2}-1}\right)
$$

where,

$$
\delta=\frac{1}{3}\left(\frac{\alpha_{\mathrm{II}}-\alpha_{\perp}}{\alpha}\right)
$$

where, $\alpha$ is the mean polarizability, $\alpha_{\text {II }}$ and $\alpha_{\perp}$ are the principal polarizabilities in direction parallel and perpendicular to optic axis, $\mathrm{S}$ is the orientational order parameter of the molecules and $\delta$ is the relative polarizability anisotropy. By using the extrapolation method of Haller [20], extrapolating the linear fit of the relation $\ln (\delta . S)$ vs. $\ln \left[1-\left(\mathrm{T} / \mathrm{T}_{\mathrm{c}}\right)\right]$ to zero temperature where $\mathrm{S}=1$, $\delta$ was calculated and then used to estimate $S$ at any desired temperature.

\begin{tabular}{|c|c|c|c|c|c|c|c|c|c|c|}
\hline \multirow{2}{*}{$\begin{array}{l}\text { Substit. } \\
\text { (X) }\end{array}$} & \multirow[t]{2}{*}{ Mixture } & \multicolumn{4}{|c|}{ Composition (mole\%) } & \multicolumn{5}{|c|}{ Transition temperature $\left({ }^{\circ} \mathrm{C}\right)$} \\
\hline & & I6 & I8 & I14 & I16 & $\mathrm{T}_{\mathrm{c}-\mathrm{A}}$ & $\mathrm{T}_{\mathrm{c}-\mathrm{N}}$ & $\mathrm{T}_{\mathrm{A}-\mathrm{N}}$ & $\mathrm{T}_{\mathrm{A}-\mathrm{I}}$ & $\mathrm{T}_{\mathrm{N}-\mathrm{I}}$ \\
\hline \multirow{3}{*}{$\mathrm{CH}_{3} \mathrm{O}$} & $\mathrm{I} 6 / \mathrm{I} 8 a$ & 46.5 & 53.5 & - & - & - & 69.5 & - & - & 77.8 \\
\hline & $\mathrm{I} 6 / \mathrm{I} 14 a$ & 61.5 & - & 38.5 & - & - & 69.0 & - & - & 73.5 \\
\hline & I6/I16a & 68.5 & - & - & 31.5 & - & - & - & - & 72.0 \\
\hline \multirow{3}{*}{$\mathrm{CH}_{3}$} & $\mathrm{I} 6 / \mathrm{I} 8 b$ & 48.5 & 51.5 & - & - & - & 39.5 & _- & - & 53.4 \\
\hline & $\mathrm{I} 6 / \mathrm{I} 14 b$ & 67.5 & - & 32.5 & _- & _- & 45.0 & _ & _ & 54.5 \\
\hline & $\mathrm{I} 6 / \mathrm{I} 16 b$ & 77.5 & - & - & 22.5 & - & 49.5 & - & - & 53.2 \\
\hline \multirow{3}{*}{$\mathrm{CN}$} & $\mathrm{I} 6 / \mathrm{I} 8 \mathrm{c}$ & 52.0 & 48.0 & - & - & - & 56.0 & - & - & 83.2 \\
\hline & $\mathrm{I} 6 / \mathrm{I} 14 \mathrm{c}$ & 62.5 & - & 37.5 & 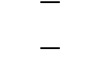 & - & 58.2 & - & - & 84.0 \\
\hline & $\mathrm{I} 6 / \mathrm{I} 16 \mathrm{c}$ & 77.5 & - & - & 22.5 & - & 60.0 & - & - & 81.5 \\
\hline \multirow{3}{*}{$\mathrm{NO}_{2}$} & I6/I8d & 53.0 & 47.0 & - & - & - & & - & 62.5 & \\
\hline & $\mathrm{I} 6 / \mathrm{I} 14 d$ & 60.0 & - & 40.0 & - & 50.0 & 39.5 & 68.5 & - & - \\
\hline & $\mathrm{I} 6 / \mathrm{I} 16 d$ & 68.5 & _- & - & 31.5 & 52.0 & - & 64.5 & - & - \\
\hline
\end{tabular}

Table (1): Mole percent of the mixtures and their transition temperatures

The estimated $\mathrm{S}$ values as function of $\left(\mathrm{T}_{\mathrm{c}} \mathrm{-T}\right)$ for each eutectic system of the binary mixtures (I6/In)X, $n=8,14,16$ and $\mathrm{X}=\mathrm{CH}_{3} \mathrm{O}(a), \mathrm{CH}_{3}(b)$, and $\mathrm{CN}$ (c), are plotted and represented in figures (5-7), respectively. The eutectic system (I6/In)d was excluded because its eutectic systems are smectogenic, see Fig. (4), which makes the comparison with the other eutectic systems, whose phases are nematogenic, be inconsistent. 
It is seen from Figs. (5-7) that irrespective of the nature of the polar group in any homologous series, the order parameter S, at any temperature, increases with the increase of the difference in number of carbon atoms between the alkoxy groups of the two components in the binary mixture. At any arbitrary temperature, $\left(\mathrm{T}_{\mathrm{c}}-\mathrm{T}\right)=4{ }^{\circ} \mathrm{C}$ for example, the order parameter $\mathrm{S}$ is plotted vs. the difference in the number of carbon atoms $(n)$ between the two homologues constituting the binary mixtures and represented in Figs. (8-10), respectively. Although, the orientational order parameter is not an additive property yet, the figures show within the experimental error a linear increase of $\mathrm{S}$ with $n$. The slope of each line, which represents the enhancement of each $\mathrm{CH}_{2}$ group difference to the order parameter, decreases as the degree of polarity of the polar group in the homologous series increases. For instance, the lowest slope value of the linear relation is observed with the homologues having the highly $\mathrm{CN}$ polar group. This is might be due to the polarity effect, since the molecules might be more tightly oriented in the field of the highly polar groups than in the field of less polar ones, which might limit the influence of the difference in the chain length of the homologues in the binary mixture.

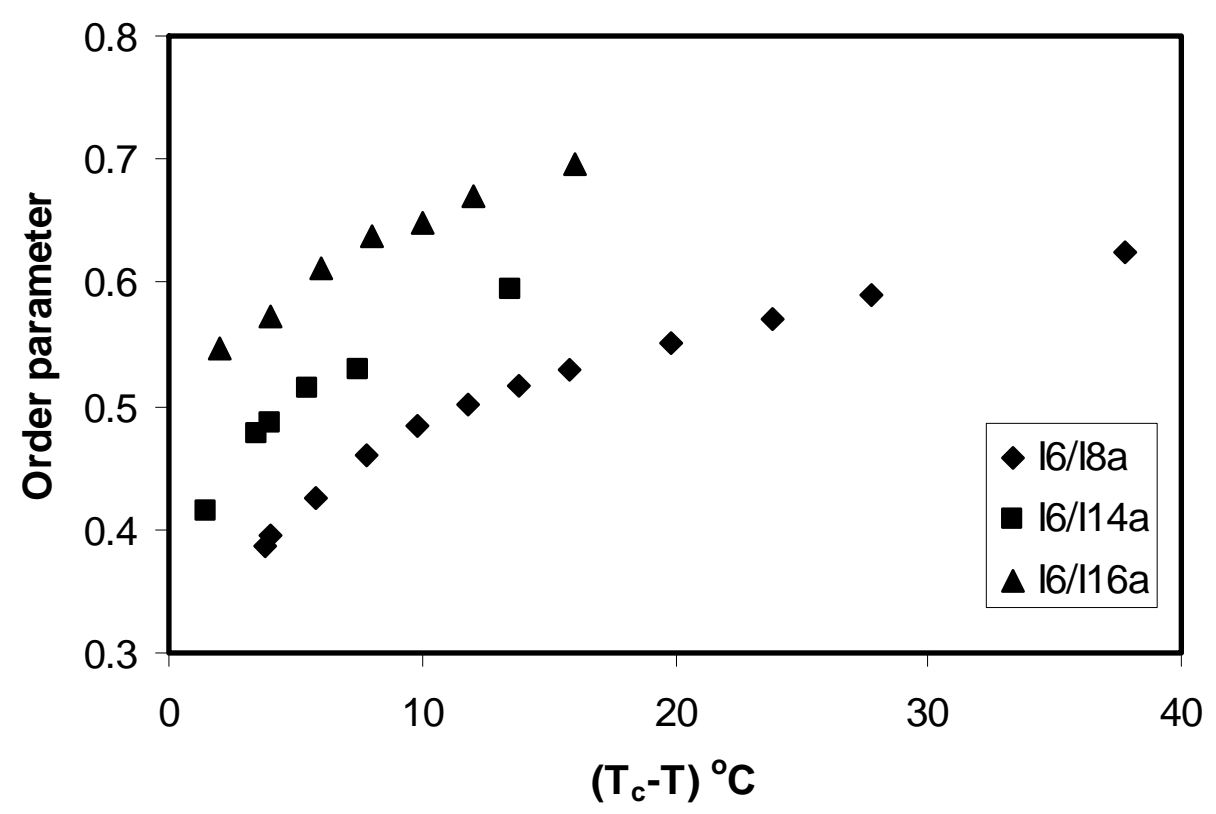

Fig. (5): Temperature dependence of the order parameter for mixtures of methoxysubstituted homologues at their eutectic compositions. 


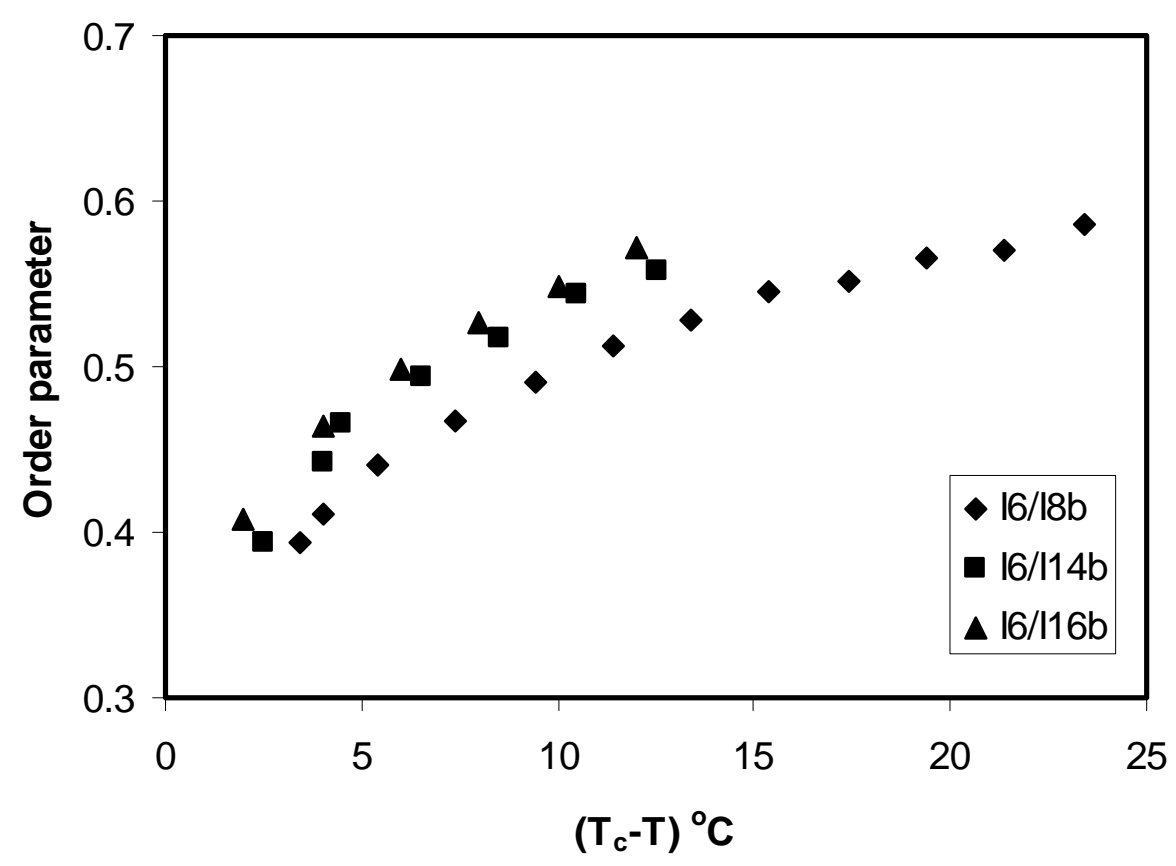

Fig. (6): Temperature dependence of the order parameter for mixtures of methylsubstituted homologues at their eutectic compositions.

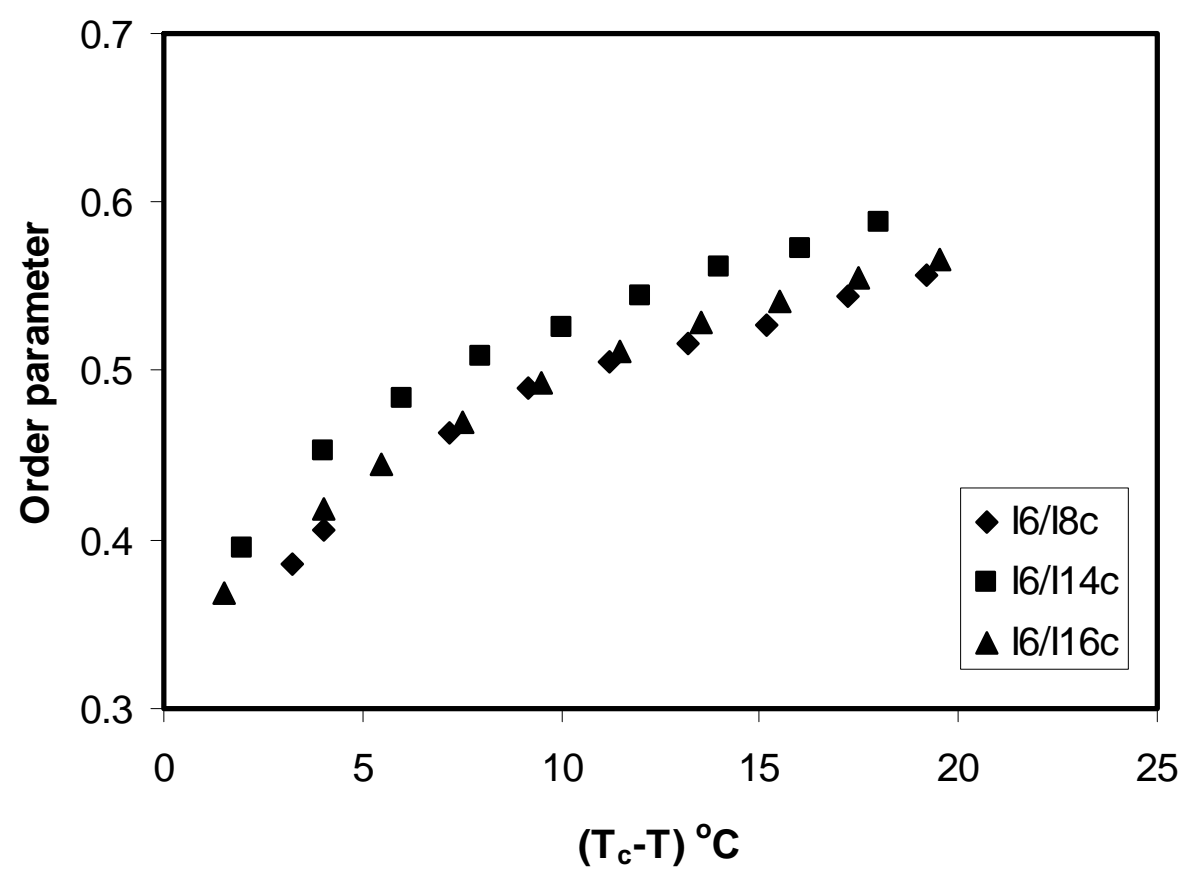

Fig. (7): Temperature dependence of the order parameter for mixtures of cyanosubstituted homologues at their eutectic compositions. 




Fig. (8): The dependence of the order parameter on the difference in carbon atoms number between the methoxy-substituted homologues in the binary mixtures at their eutectic compositions.

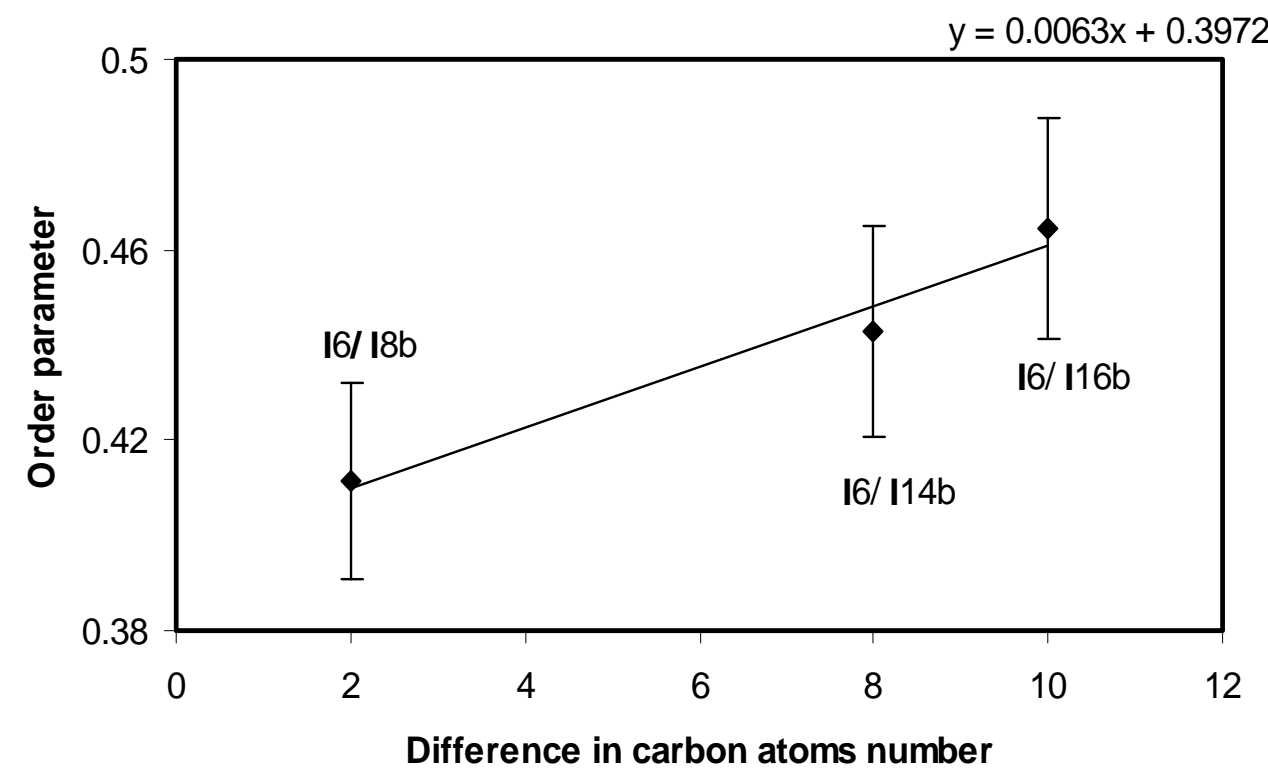

Fig. (9): The dependence of the order parameter on the difference in carbon atoms number between the methyl-substituted homologues in the binary mixtures at their eutectic compositions. 


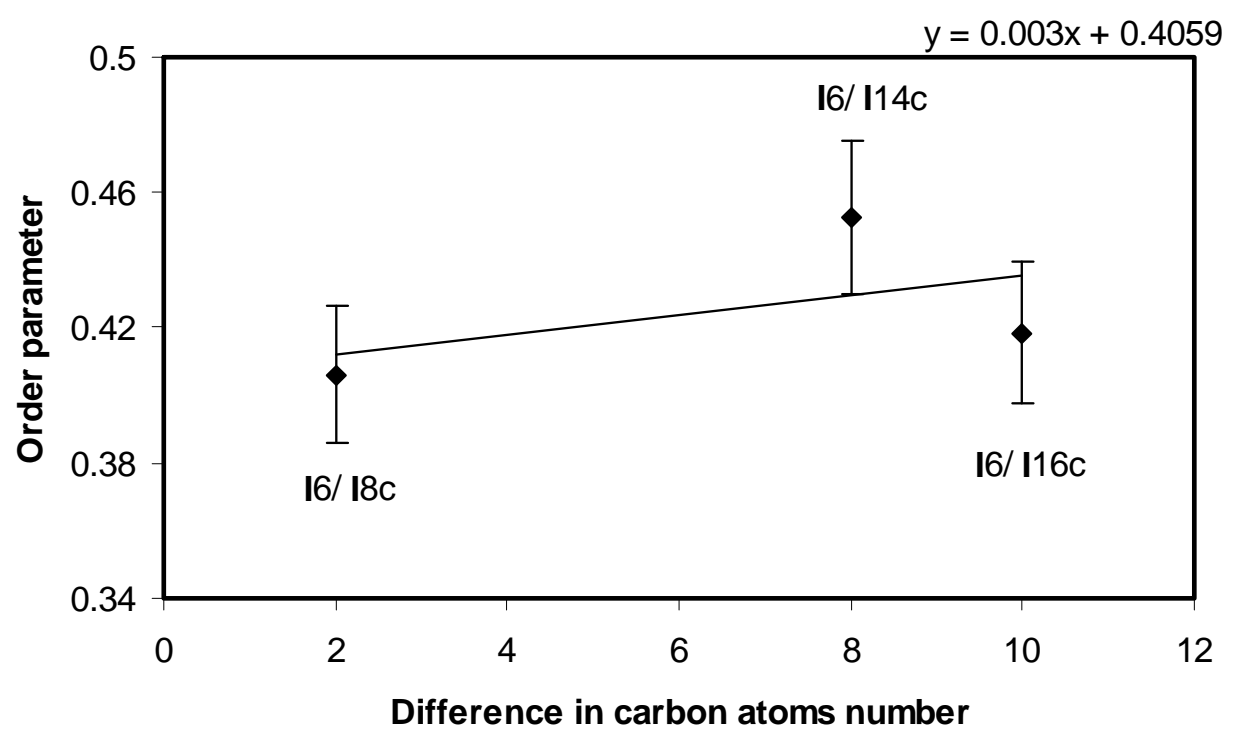

Fig. (10): The dependence of the order parameter on the difference in carbon atoms number between the cyano-substituted homologues in the binary mixtures at their eutectic compositions.

\section{Conclusion:}

Twenty-four phase diagrams of the all possible binary mixtures of the liquid crystalline esters $\operatorname{In}(a-d)$ were constructed in order to investigate the effect of the chain length of the alkoxy group on the thermal phase behaviour. From the phase diagrams and the Fig. (5-10), the following conclusions would be obtained:

1- With respect to the binary mixtures of homologues bearing electronreleasing substituents Ina and Inb, all the systems tended to possess eutectic composition irrespective of the difference between chain lengths of the two components in the binary system. Since the methyl group is weak electronreleasing compared with the methoxy one, the melting point of their eutectic composition is much more depressed and accordingly led to the appearance of a wider composition range of nematic phase.

2- With respect to binary mixtures of homologues bearing electronwithdrawing substituents Inc and Ind, since I $6 c$ and I $8 c$ are enantiotropically nematogenic while the higher homologues are smectogenic, the I6c/I8c binary mixtures show only nematic phase, whereas the smectic phase starts to appear from I $6 c / \mathrm{I} 14 c$ and increases through the other mixtures $\mathrm{I} 6 c / \mathrm{I} 16 c$, $\mathrm{I} 8 \mathrm{c} / \mathrm{I} 14 \mathrm{c}$ and $\mathrm{I} 8 \mathrm{c} / \mathrm{I} 16 \mathrm{c}$ to cover the whole composition range in the I14c/I16c. With respect to Ind, since the lower homologue I6d is monotropically nematic while I8d possesses smectic-A and nematic phases, 
I6d/I8d binary mixture showed a wide nematic range. The other two systems I6d/I14d and I6d/I16d possess smecticA phase starting from about $20 \mathrm{~mole} \%$ of I14d or I16d respectively. The other extreme appears in the I14d/I16 $d$ mixture where its whole composition range is purely smectogenic.

3- The estimated order parameter $\mathrm{S}$ for the eutectic compositions of the mixtures $\operatorname{I} 6 / \operatorname{In}(a-d)$ was found to increase as the difference in chain length between the alkoxy groups of the components in the binary mixture increases, irrespective of the nature of the polar group of homologous series except the binary mixture I6c/I16c which showed a different behavior. However, within the experimental error, the S-dependence of each one of the homologous series on the difference in number of carbon atoms, $n$, of the alkoxy groups at some fixed temperature, showed a linear relation. The slope of each line depends on the nature of the polar group such that it decreases as the strength of the polar group increases.

\section{Acknowledgement}

The author would like to appreciate the helpful discussion of Prof. Dr. M. M. Naoum, Dept. of Chemistry, Faculty of Science, University of Cairo, Egypt.

\section{References}

1. G. W. Gray, "Molecular Structure and Properties of Liquid Crystals", New York, Academic Press (1962).

2. H. Sackmann, and D. Demus, Z. Phys. Chem., 224, 177; (1963), Z. Phys. Chem., 230, 285 (1965).

3. J. S. Dove, P. R. Patel, and K. L. Vasnath, Indian J. Chem., 4, 505 (1966). Mol. Cryst. Liq. Cryst., 8, 93 (1969).

4. M. M. Naoum, R. I.Nessiem, G. R. Saad, and T. Y. Labeeb, Liq. Cryst., 29, 929 (2002).

5. R. I. Nessim, M. M. Naoum, and M. Nessim, Liq. Cryst. (in press).

6. M. M. Naoum, R. I. Nessim, G., R. Saad, and T. Y. Labeeb, Liq. Cryst., 29, 229 (2002).

7. R., I. Nessim, J. Therm. Anal. Calri. (in press).

8. P. Palffy-Muhoray, D., A. Balzarini, and D., A. Dunmur, Mol. Cryst. Liq. Cryst., 110, 315 (1984).

9. Y. Narashimha Murthy, V. Rama Murthy, and R. Ranga Reddy, N., V., Cryst. Res. Technol., 32, 279 (1997).

10. P. Adamsky, Cryst. Res. Technol., 34, 763 (1999). 
11. M. Roushdy, Liq. Cryst., 31, 371 (2004).

12. H. J. Müller, and W. Haase, Mol. Cryst. Liq. Cryst., 409, 127 (2004).

13. A. Ghanadzadeh, H. Ghanadzadeh, and N. Khshroo, J. Mol. Liq., 94, 37 (2001)

14. M. Roushdy, Mol. Cryst. Liq. Cryst. 457, 151 (2006).

15. D. Sunanda, M. S. Madhava, D. Revannasiddaiah, R. Somashekar, and J. Mahadeva, Mol. Cryst. Liq. Cryst., 409, 163 (2004).

16. M. Roushdy, Mol. Cryst. Liq. Cryst., 461, 53 (2007).

17. R., I. Nessim, Therm. Chem. Acta 389, 49(2002).

18. V. G. Chigrinov, "Liquid crystal devices: physics and applications", Artec House (Boston-London), Chapt. 1, p. 33 (1999).

19. M. F. Vuks, Optics and Spectroscopy, 20, 361 (1966).

20. I. Haller, M., A. Huccins, M., R. Lilienthal, and T. R. Mc Cuire, J. Phys. Chem., 77, 950 (1973). 